BULL. AUSTRAL. MATH. SOC.

MOS 2008

VOL. I (1969), 417-418.

\title{
Some varieties without the amalgam embedding property
}

\author{
Stephen Meskin
}

\begin{abstract}
A variety of groups $V$ has the amalgam embedding property if every amalgam of two $\underline{\underline{V}}$-groups can be embedded in a $\underline{\underline{V}}$-group. In this note the author proves that if $\underline{\underline{V}}$ is a variety of exponent 0 which satisfies a law $W\left(x_{1}{ }^{n}, x_{2}, \ldots, x_{t}\right)$ but not $W\left(x_{1}, x_{2}, \ldots, x_{t}\right)$ then $\underline{\underline{v}}$ does not have the amalgam embedding property.
\end{abstract}

A variety of groups $\underline{\underline{V}}$ has the amalgam embedding property (AEP) if every amalgam of two $\underline{\underline{V}}$-groups can be embedded in a $\underline{\underline{V}-g r o u p . ~ N o ~ v a r i e t i e s ~}$ other than the variety of all groups and abelian varieties are known to have AEP . (See [1] pp. $42-43$ for references and comments on this problem).

In this note we prove the following:

THEOREM. If $\underline{\underline{V}}$ is a variety of exponent 0 which satisfies a law $W\left(x_{1}{ }^{n}, x_{2}, \ldots, x_{t}\right)$ but not $W\left(x_{1}, x_{2}, \ldots, x_{t}\right)$ then $\underline{\underline{v}}$ does not have AEP .

Proof. Let $F$ be the free V-group on $t$ generators $y_{1}, y_{2}, \ldots, y_{t}$; then $g p\left(y_{1}\right)$ is infinite cyclic and $W\left(y_{1}, y_{2}, \ldots, y_{t}\right) \neq 1$, for every relator on the free generators of $F$ is a law in $\underline{\underline{V}}$.

Let $Z$ be the infinite cycle generated by $z$. Certainly $Z$ is a

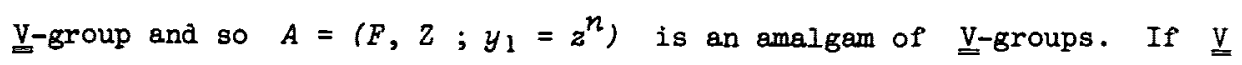

Received 30 July 1969. 


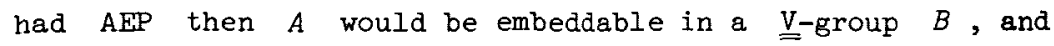

$W\left(y_{1}, y_{2}, \ldots, y_{t}\right)=W\left(z^{n}, y_{2}, \ldots, y_{t}\right)=1$ in $B$, which is a contradiction.

\section{Reference}

[1] Hanna Neumann, Varieties of groups, (Ergebnisse der Mathematik, und ihrer Grenzgebiete, Band 37, Springer-Verlag, Berlin, Heidelberg, New York, 1967).

Department of Mathematics, IAS, Australian National University, Canberra, ACT. 